\title{
The Value of MicroRNAs as an Indicator of the Severity and the Acute Phase of Spinal Cord Injury
}

\author{
Junyoung Park, MD, Dongsoo Yi, MD, Jiyoon Jang, MD, Jiseon Hong, MD
}

Department of Rehabilitation Medicine, Wonju Severance Christian Hospital, Yonsei University Wonju College of Medicine, Wonju, Korea

Objective To assess the role of miRNA-21 and miRNA-223 in a balloon-compression model of spinal cord injury (SCI). Methods A total of 50 male Wistar rats $(n=50)$ were divided into the three groups: the group A $(n=15$, insertion of the unflated Fogarty balloon catheter), the group B ( $n=15$, insertion of the Fogarty balloon catheter at a volume of $20 \mu \mathrm{L})$ and the group $\mathrm{C}(\mathrm{n}=15$, insertion of the Fogarty balloon catheter at a volume of $50 \mu \mathrm{L})$. After the behavioral test, RNA isolation, microRNA expression profiling using microarrays and quantitative polymerase chain reaction, measurements were compared between the three groups.

Results Despite a lack of significant differences in time-dependent changes in miRNA-21 expression levels between the three groups at 4 hours, there were significant differences in them at 1,3 , and 7 days $(p<0.05)$. Moreover, there were significant differences in time-dependent changes in miRNA-223 expression levels between the three groups at 4 hours and 1, 3, and 7 days $(\mathrm{p}<0.05)$. Furthermore, miRNA-223 expression levels reached the highest at 1 day but were decreased with time thereafter in all the three groups.

Conclusion Expression levels of miRNA-21 and miRNA-223 might be associated with the severity and acute phase of SCI, respectively. It is mandatory, however, to analyze changes in levels of inflammatory markers and the relevant biological pathways.

Keywords Spinal cord, Spinal cord injuries, MicroRNAs, Gene expression regulation, Therapeutics

\section{INTRODUCTION}

Spinal cord injury (SCI) is a devastating event that arises from trauma to the vertebra; it is characterized by mechanical disruption of the spinal cord [1]. Current treatment modalities are effective only in a limited scope.
Only acute methylprednisolone therapy has had protective effects on SCI [2]. The SCI is considered a serious health problem that may impair the quality of life in affected individuals [3]. It is therefore imperative that new therapeutic strategies be established for the treatment of patients with SCI for which its cellular and molecular

Department of Rehabilitation Medicine, Wonju Severance Christian Hospital, Yonsei University Wonju College of Medicine, 20 Ilsan-ro, Wonju 26426, Korea. Tel: +82-33-741-1836, Fax: +82-33-741-1455, E-mail: sadocean17@naver.com

ORCID: Junyoung Park (http://orcid.org/0000-0001-5721-4112); Dongsoo Yi (http://orcid.org/0000-0002-5764-0610); Jiyoon Jang (http://orcid. org/0000-0002-7635-8901); Jiseon Hong (http://orcid.org/0000-0002-7412-2145).

(c) This is an open-access article distributed under the terms of the Creative Commons Attribution Non-Commercial License (http://creativecommons.org/ licenses/by-nc/4.0) which permits unrestricted noncommercial use, distribution, and reproduction in any medium, provided the original work is properly cited. Copyright $\odot 2019$ by Korean Academy of Rehabilitation Medicine 
pathophysiology should be further explored.

A better understanding of the pathophysiological mechanisms underlying the onset of SCI is essential for developing effective therapeutic approaches; its key events include injury and involvement of various factors resulting in the functional deficits. Thus, its pathophysiology is well described as a biphasic process; it consists of primary and secondary phase of injury. The primary phase of injury involves the initial mechanical impact that is characterized by exertion of the force to the spinal cord and the disruption of axons, blood vessels, and cell membranes. This is followed by the secondary phase of injury that is characterized by inflammation and delayed apoptotic events. Despite the immediate presence of neurological deficits following the onset of initial injury, the secondary phase of injury leads to a prolonged period of tissue destruction [4]. Thus, worsening of SCI is closely associated with its secondary pathophysiology, which leads to the extension of the paralysis to higher spinal segments. Patients with SCI are therefore vulnerable to paralysis due to inhibition of the generation of neuronal precursor cells after several years of persistence of such pathophysiological changes $[5,6]$.

Over the past decade, many clinical and translational studies have been conducted to propose new treatment strategies. Ongoing studies focus on factors involved in the secondary pathophysiology of SCI, thus attempting to promote regeneration and replace destroyed spinal cord tissue [7].

Studies about complex interaction between the cellular and molecular pathophysiologic events of SCI have broadened the understanding of it. Next, inhibition of multiple pathogenic mechanisms and promotion of neuroregeneration should be further studied in this series. Moreover, the severity of SCI is known as a strong prognostic indicator that is closely associated with the neurologic grade on admission in patients with SCI [8].

MicroRNA (miRNA) sequences have a hairpin-like structure, and they are small, unique, non-coding RNA fragments with a mean length of 22 nucleotides. Their potential roles in regulating biological pathways underlying the pathophysiology of SCI have been described in the literature. Thus, they are involved in neurogenesis and cortical development [9].

To date, more than 550 miRNAs have been identified from mammalian cells and their biological roles have been well documented. Of these, several miRNAs such as miRNA-1, miRNA-10a, miRNA-338, miRNA-451, miRNA34a, miRNA-133, miRNA-142-3p, miRNA-199, miRNA$10 \mathrm{~b}$ and miRNA-219 are abundantly present in the spinal cord. This is accompanied by previous published studies showing that their up-regulation or down-regulation are associated with the onset of SCI [10-17]. Moreover, identical families of miRNAs might target the same categories of genes. It can therefore be inferred that miRNAs are concurrently involved in the regulation of specific physiological processes underlying the onset of SCI $[15,16]$.

The SCI is characterized by two molecular events such as inflammatory responses and apoptotic events. First, the pathogenesis of both acute and chronic SCI is regulated by inflammatory responses that might play a key role in the onset of nerve injury and the control of regenerative responses. Second, apoptotic events of neurons and oligodendrocytes leading to impaired neuronal functions greatly contribute to the paralysis of patients with SCI [18]. From this context, both miRNA-21 and miRNA-223 are closely associated with the secondary pathophysiology of SCI; experimental studies have shown that they are involved in the apoptosis and the acute phase of inflammation $[10,19]$.

We have therefore speculated that there would be dramatic improvements in the severity and symptoms of SCI if it becomes possible to clarify the roles of miRNA-21 and miRNA-223 in association with its onset.

Given the above background, we conducted this experimental study to assess the role of miRNA-21 and miRNA-223 in an animal model of SCI and to discuss their clinical and therapeutic implications. To do this, we monitored time-dependent changes in their expression levels. There is an emerging evidence that there are timedependent changes in RNA expression in the spinal cord and this may be critical in the progression of SCI $[20,21]$.

\section{MATERIALS AND METHODS}

\section{Ethics statement}

The current study was approved by the Institutional Review Board of Wonju Severance Christian Hospital (no. CR318049). All the laboratory procedures were performed in compliance with the revised guidelines of the US National Institutes of Health. 


\section{Experimental animals}

For the current laboratory procedure, we housed 50 male Wistar rats $(n=50)$, aged 8 weeks, weighing $300-320 \mathrm{~g}$, in the animal facility with 12-hour light/dark cycles. The temperature of the facility was maintained at $25^{\circ} \mathrm{C}-28^{\circ} \mathrm{C}$. The rats were allowed for free access to water and standard rat chow.

\section{An animal model of SCI}

We used a balloon-compression model of SCI as previously described [22]. The experimental rats were anesthetized using 3\% isoflurane (Terrell; Piramal Critical Care Inc., Bethlehem, PA, USA). Then, the anesthesia was maintained with $2.5 \%$ isoflurane. The experimental rats were placed in sternal recumbency and the lumbosacral region was shaved accordingly. This was followed by the treatment with povidone and alcohol.

Of the experimental animals, 45 were randomized to three groups: group $A(n=15$, insertion of the unflated Fogarty balloon catheter), group B ( $n=15$, insertion of the Fogarty balloon catheter at a volume of $20 \mu \mathrm{L}$ ), and group C $(n=15$, insertion of the Fogarty balloon catheter at a volume of $50 \mu \mathrm{L}$ ).

An animal model of SCI was established under the fluoroscopic guidance (MCA-6100 mobile C-arm system; Medison Xray Inc., Seoul, Korea) for which the experimental rats underwent insertion of the BD Perisafe 18 G $\times 5$ " Weiss Epidural Needle with fixed wings, Modified Tuohy Point (BD, Franklin Lakes, NJ, USA) in the lumbosacral joint. This was followed by placement of a 2-F Fogarty balloon catheter (Edwards Fogarty; Edwards Lifesciences, Irvine, CA, USA) using the spinal needle in the epidural space. The 2-F Fogarty balloon catheter was filled with iohexol with saline diluted at a ratio of 1:1 (Omnipaque; Amersham Health, Cork, Ireland) and then connected to a 50- $\mu \mathrm{L}$ Hamilton syringe (type 1705; Hamilton Company, Reno, NV, USA). The tip of the 2-F Fogarty balloon catheter was placed in the 9th thoracic spine and then inflated to the volume of 20 and $50 \mu \mathrm{L}$ for 10 minutes using iohexol with saline diluted at a ratio of 1:1. After confirming the location and shape of the balloon catheter, we deflated and then removed it. This was followed by urinary bladder maintenance at a frequency of two times/day without treatment with antibiotics.

An animal model of SCI was validated on behavioral assessment and histopathological findings. Prior to sac- rifice, gait and locomotor functions of the experimental rats were assessed using the Basso, Beattie and Bresnahan (BBB) locomotor scale, ranging from 0 (no locomotor activity) to 21 (normal function) as previously described [23]. Thus, the BBB scores were plotted as functions of the length of time (at 3 hours and 1, 3, 5, and 7 days).

\section{RNA isolation}

Total RNA was extracted from the parenchyma of the spinal cord using the TRIzol reagent (Invitrogen, Carlsbad, CA, USA), and its concentration and quantity were measured based on the UV absorbance at a wavelength of 260 and $280 \mathrm{~nm}$ (A260/280) and then confirmed using a gel electrophoresis. The RNA sample was used for the quantitative polymerase chain reaction (PCR).

\section{Quantitative PCR}

For the quantitative PCR, we used a TaqMan miRNA assay kit (Applied Biosystems, Foster City, CA, USA). Then, we performed reverse transcription reactions for the mature miRNA containing a sample of total RNA, 50 nM stem-loop RT primer, 10× RT buffer, $100 \mathrm{mM}$ of each $\mathrm{dNTP}, 50 \mathrm{U} / \mu \mathrm{L}$ MultiScribe Reverse Transcriptase and 20 $\mathrm{U} / \mu \mathrm{L}$ RNase inhibitor. This was followed by incubation of reaction mixtures $(15 \mu \mathrm{L})$ in a thermal cycler (Rotor-Gene Q; Qiagen, Hilden, Germany) for 30 minutes at $16^{\circ} \mathrm{C}, 30$ minutes at $42^{\circ} \mathrm{C}$, and 5 minutes at $85^{\circ} \mathrm{C}$. Then, the reaction was maintained at $4^{\circ} \mathrm{C}$.

The real-time PCR (RT-PCR) was performed using the Rotor-Gene Q instrument with a $10 \mu \mathrm{L}$ of the PCR mixture containing a $1.33 \mu \mathrm{L}$ of the RT-PCR product, $2 \times$ TaqMan Universal PCR Master Mix, $0.2 \mu \mathrm{M}$ TaqMan probe, $15 \mu \mathrm{M}$ forward primer, and $0.7 \mu \mathrm{M}$ reverse primer. All the reaction mixtures were incubated in duplicate in a 72 -well rotor at $95^{\circ} \mathrm{C}$ for 10 minutes, followed by 40 cycles of $95^{\circ} \mathrm{C}$ for 15 seconds and $60^{\circ} \mathrm{C}$ for 1 minute. The U6 served as a control to normalize differences in total RNA levels between the samples. Following analysis of a threshold cycle (Ct) in the exponential phase of amplification, relative expression levels were quantified using standard curves for target genes and the endogenous control. Following calculation of the $\Delta \Delta \mathrm{Ct}$ values using geometric means, thus expressed as $2^{-\Delta \Delta \mathrm{Ct}}$, the $\Delta \Delta \mathrm{Ct}$ value of each control sample was set at 1 . Thus, changes in relative expression levels of target genes were analyzed. 


\section{Statistical analysis}

For statistical analysis, all data was expressed as mean \pm standard deviation. Data analysis was performed using the SPSS version 18.0 for Windows (SPSS Inc., Chicago, IL, USA). Differences in measurements between the three experimental groups were tested for statistical significance using the repeated measures analysis of variance (ANOVA) and Duncan's post-hoc analysis. A p-value of $<0.05$ was considered statistically significant.

\section{RESULTS}

Validation of an animal model of $\mathrm{SCl}$ based on results of behavioral assessment

There were no time-dependent changes in the BBB scores in the group A. But the group B and C showed significant time-dependent changes in them $(\mathrm{p}<0.05)$ (Fig. 1).

Time-dependent changes in miRNA-21 expression levels

Time-dependent changes in miRNA-21 expression

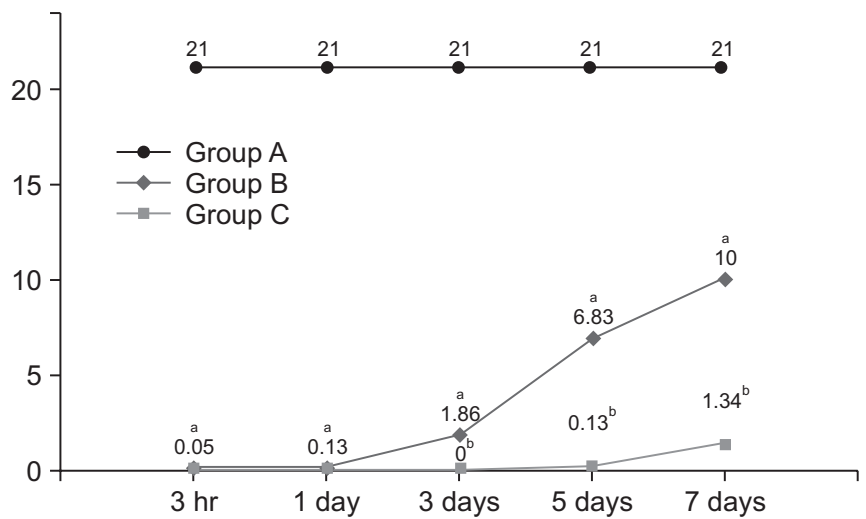

Fig. 1. Time-dependent changes in the Basso, Beattie and Bresnahan (BBB) scores. There were significant timedependent increases in the BBB scores in both the group $B$ and C $(p<0.05)$. Different letters indicate statistical significance $(\mathrm{p}<0.05)$.

Table 1. Time-dependent changes in the level of miRNA-21 expression

\begin{tabular}{lrrr}
\hline Time point & Group A & Group B & Group C \\
\hline 4 hours & $1.27 \pm 0.22$ & $1.36 \pm 0.40$ & $1.12 \pm 0.23$ \\
\hline day & $1.88 \pm 0.24$ & $3.80 \pm 1.16$ & $5.31 \pm 2.06$ \\
\hline days & $0.02 \pm 0.18$ & $4.43 \pm 1.14$ & $8.50 \pm 1.50$ \\
7 days & $2.63 \pm 1.31$ & $5.28 \pm 1.50$ & $10.16 \pm 0.95$ \\
\hline
\end{tabular}

Values are presented as mean \pm standard deviation and $2^{-\Delta \Delta \mathrm{Ct}}(\mathrm{Ct}$, threshold cycle). levels are shown in Table 1 and Fig. 2. At 4 hours, there were no significant differences in them between the three groups. At 1, 3, and 7 days, however, there were significant differences in them between the three groups $(p<0.05)$. These results indicate that miRNA-21 expression levels might serve as an indicator of the severity of SCI.

Time-dependent changes in miRNA-223 expression levels Time-dependent changes in miRNA-223 expression levels are shown in Table 2 and Fig. 3. At 4 hours and 1, 3, and 7 days, there were significant differences in them between the three groups $(\mathrm{p}<0.05)$. Moreover, miRNA-223 expression levels reached the highest at 1 day but were decreased with time thereafter in all the three groups. These results indicate that miRNA-223 expression levels might serve as an indicator of the acute phase of SCI.

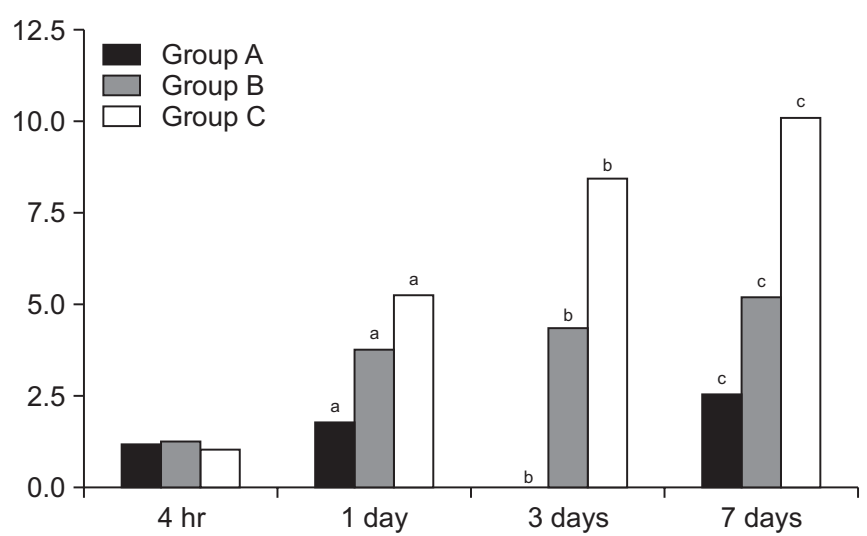

Fig. 2. Time-dependent changes in the level of miRNA-21 expression. Despite a lack of significant differences in time-dependent changes in miRNA-21 expression levels between the three groups at 4 hours, there were significant differences in them at 1,3 , and 7 days $(\mathrm{p}<0.05)$. Different letters indicate statistical significance $(p<0.05)$. The y-axis represents $2^{-\Delta \Delta C t}(\mathrm{Ct}$, threshold cycle).

Table 2. Time-dependent changes in the level of miRNA-223 expression

\begin{tabular}{lrrr}
\hline Time point & Group A & Group B & Group C \\
\hline 4 hours & $3.07 \pm 3.02$ & $8.47 \pm 4.05$ & $12.82 \pm 2.06$ \\
\hline day & $5.35 \pm 1.13$ & $14.31 \pm 4.23$ & $26.88 \pm 7.45$ \\
\hline days & $1.41 \pm 0.38$ & $3.48 \pm 0.75$ & $11.01 \pm 2.20$ \\
7 days & $1.37 \pm 0.68$ & $2.67 \pm 1.33$ & $6.48 \pm 0.99$ \\
\hline
\end{tabular}

Values are presented as mean \pm standard deviation and $2^{-\Delta \Delta \mathrm{Ct}}$ (Ct, threshold cycle). 


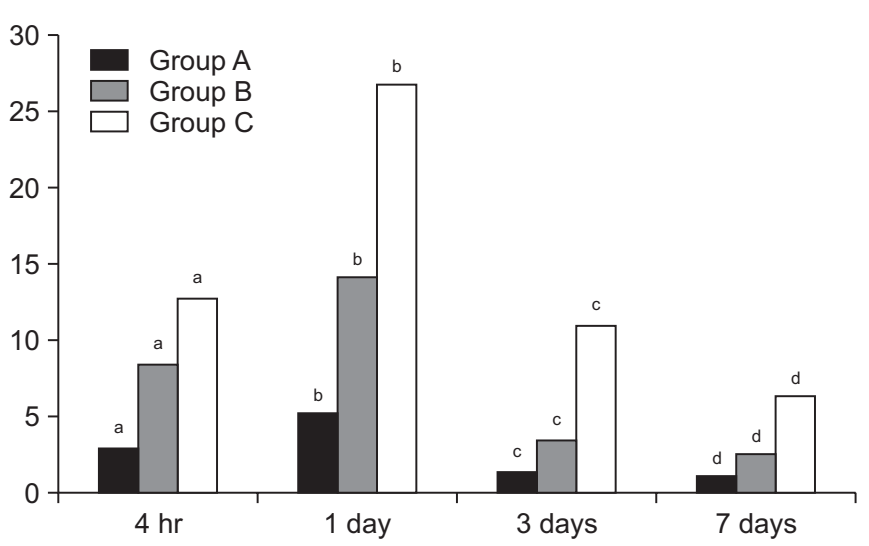

Fig. 3. Time-dependent changes in the level of miRNA-223 expression. There were significant differences in time-dependent changes in miRNA-223 expression levels between the three groups at 4 hours and 1, 3, and 7 days $(\mathrm{p}<0.05)$. Moreover, miRNA-223 expression levels reached the highest at 1 day but were decreased with time thereafter in all the three groups. Different letters indicate statistical significance $(\mathrm{p}<0.05)$. The $\mathrm{y}$-axis represents $2^{-\Delta \Delta \mathrm{Ct}}(\mathrm{Ct}$, threshold cycle).

\section{DISCUSSION}

To date, considerable efforts have been made to clarify the pathophysiology of SCI. This has contributed to the development of pharmacologic and cell-based therapeutic approaches, which is accompanied by animal models showing a functional motor recovery. Of these, several therapeutics have also been tested in clinical trials [24].

It is generally believed that SCI is an incurable disease leading to permanent disabilities. But there is a possibility that it might be treated with some therapeutic regimens as shown in studies about the efficacy of pharmacological agents and hypothermia [25]. Methylprednisolone is an effective neuroprotective agent, but its adverse effects remain a great concern. Hypothermia is also an effective neuroprotective modality, but its effectiveness deserves further evidence-based clinical studies. In addition, neuroregenerative approaches are also attempted for the treatment of SCI. They are advantageous in that they are improving endogenous regenerative potentials without adverse effects. Recent emergence of cell-based therapies has been of increasing interest as a neurotrophic support for the treatment of SCI [26].

Despite promising results of clinical studies in this series, there is still a lack of gold standard. It would therefore be mandatory to conduct future studies focusing on both neuroprotective and neuroregenerative approaches [27].

For the current experimental study, we used a ballooncompression model of SCI; we applied three different volumes to the rats and thereby successfully created an animal model of SCI with a severity ranging from a nearnormal condition to a severe one. This was demonstrated on behavioral test showing significant differences in BBB scores between the three groups. It can therefore be inferred that a balloon-compression model of SCI; its functional outcomes may have a correlation with the degree of stimulus applied to the rats [28].

In the current study, despite a lack of significant differences in time-dependent changes in miRNA-21 expression levels between the three groups at 4 hours, there were significant differences in them at 1,3 , and 7 days. These results indicate that miRNA-21 expression levels might serve as an indicator of the severity of SCI. Of note, up-regulation of miRNA-21 was associated with several types of CNS injuries, such as traumatic brain injury and brain ischemia $[29,30]$. This is accompanied by published studies showing that the dysregulation of miRNA-21 was involved in the pathogenesis of SCI in a rat SCI model $[14,15]$. Taken together, it can be inferred that miRNA-21 expression might be associated with the onset of SCI. Still, however, little is known about its association with the severity of SCI.

We also found that there were significant differences in time-dependent changes in miRNA-223 expression levels between the three groups at 4 hours and 1, 3, and 7 days. Moreover, miRNA-223 expression levels reached the highest at 1 day but were decreased with time thereafter in all the three groups. These results indicate that the level of miRNA-223 expression might be an indicator of the acute phase of SCI. This is in agreement with a previous report; Izumi et al. [19] showed that there was upregulation of miRNA-223 in the early phase of secondary damage after the onset of SCI.

Limitations of the current study are as follows. (1) We failed to analyze inflammatory markers that are indicative of the severity of SCI. To date, considerable efforts have been made to identify inflammatory markers in this series. Thus, it has been suggested that a series of inflammatory markers (IL-6, IL-8, and MCP-1) and structural proteins (tau, S100 $\beta$ and GFAP) are associated with the severity of SCI [31-33]. This is based on previous pub- 
lished studies showing that there is a correlation between levels of inflammatory cytokines after the onset of SCI and its severity [34,35]. (2) We failed to analyze biological pathways associated with the severity of SCI. According to Sengupt et al. [36], of the 49 proteins isolated from human cerebrospinal fluid samples, 8 showed a differential expression. These authors suggested that these proteins are involved in several molecular pathways underlying a set of pathophysiologic mechanisms depending on the severity of SCI in response to the assault. (3) There is a discrepancy in SCI between experimental models and a clinical settings. That is, animal models of SCI are characterized by damages to the thoracic spine and they occur dorsally. But human SCI occurs anteriorly and it is characterized by frequent damages to the cervical spine. Moreover, there is a close relationship between the location of lesions and the pathology and degree of SCI. That is, the anterior spinal artery and the dorsal one are affected in humans and animal models, respectively. The former is responsible for the blood supply for $3 / 4$ of the spinal cord tissue $[37,38]$. It would therefore be mandatory to reflect the severity of SCI as closely as possible to clinical settings in establishing animal models of it.

In conclusion, expression levels of miRNA-21 and miRNA-223 might be associated with the severity and acute phase of SCI, respectively. But further studies are warranted to analyze changes in levels of inflammatory markers and the relevant biological pathways.

\section{CONFLICT OF INTEREST}

No potential conflict of interest relevant to this article was reported.

\section{AUTHOR CONTRIBUTION}

Conceptualization: Park J, Yi DS, Jang J, Hong J. Methodology: Park J. Formal analysis: Park J, Jang J. Project administration: Yi DS, Hong J. Visualization: Park J, Hong J. Writing - original draft: Park J, Yi DS. Writing - review and editing: Park J, Yi DS. Approval of final manuscript: all authors.

\section{REFERENCES}

1. Anwar MA, Al Shehabi TS, Eid AH. Inflammogenesis of secondary spinal cord injury. Front Cell Neurosci 2016;10:98.

2. Bracken MB. Steroids for acute spinal cord injury. Cochrane Database Syst Rev 2012;1:CD001046.

3. Budh CN, Osteraker AL. Life satisfaction in individuals with a spinal cord injury and pain. Clin Rehabil 2007;21:89-96.

4. Rowland JW, Hawryluk GW, Kwon B, Fehlings MG. Current status of acute spinal cord injury pathophysiology and emerging therapies: promise on the horizon. Neurosurg Focus 2008;25:E2.

5. Xu J, Kim GM, Chen S, Yan P, Ahmed SH, Ku G, et al. iNOS and nitrotyrosine expression after spinal cord injury. J Neurotrauma 2001;18:523-32.

6. Schaefer A, O'Carroll D, Tan CL, Hillman D, Sugimori $\mathrm{M}$, Llinas $\mathrm{R}$, et al. Cerebellar neurodegeneration in the absence of microRNAs. J Exp Med 2007;204:1553-8.

7. Carlson GD, Gorden C. Current developments in spinal cord injury research. Spine J 2002;2:116-28.

8. Silberstein M, Tress BM, Hennessy O. Prediction of neurologic outcome in acute spinal cord injury: the role of CT and MR. AJNR Am J Neuroradiol 1992;13:1597-608.

9. Yilmaz T, Kaptanoglu E. Current and future medical therapeutic strategies for the functional repair of spinal cord injury. World J Orthop 2015;6:42-55.

10. Hu JZ, Huang JH, Zeng L, Wang G, Cao M, Lu HB. Anti-apoptotic effect of microRNA-21 after contusion spinal cord injury in rats. J Neurotrauma 2013; 30:1349-60.

11. Bhalala OG, Srikanth M, Kessler JA. The emerging roles of microRNAs in CNS injuries. Nat Rev Neurol 2013;9:328-39.

12. Hu JR, Lv GH, Yin BL. Altered microRNA expression in the ischemic-reperfusion spinal cord with atorvastatin therapy. J Pharmacol Sci 2013;121:343-6.

13. Jee MK, Jung JS, Im YB, Jung SJ, Kang SK. Silencing of miR20a is crucial for Ngn1-mediated neuroprotection in injured spinal cord. Hum Gene Ther 2012;23:50820.

14. Liu NK, Wang XF, Lu QB, Xu XM. Altered microRNA expression following traumatic spinal cord injury. Exp Neurol 2009;219:424-9.

15. Strickland ER, Hook MA, Balaraman S, Huie JR, Grau JW, Miranda RC. MicroRNA dysregulation following spinal cord contusion: implications for neural plasticity and repair. Neuroscience 2011;186:146-60. 
16. Yunta M, Nieto-Diaz M, Esteban FJ, Caballero-Lopez M, Navarro-Ruiz R, Reigada D, et al. MicroRNA dysregulation in the spinal cord following traumatic injury. PLoS One 2012;7:e34534.

17. Zhou S, Ding F, Gu X. Non-coding RNAs as emerging regulators of neural injury responses and regeneration. Neurosci Bull 2016;32:253-64.

18. Yip PK, Malaspina A. Spinal cord trauma and the molecular point of no return. Mol Neurodegener 2012;7:6.

19. Izumi B, Nakasa T, Tanaka N, Nakanishi K, Kamei N, Yamamoto R, et al. MicroRNA-223 expression in neutrophils in the early phase of secondary damage after spinal cord injury. Neurosci Lett 2011;492:114-8.

20. Cheng X, Fu R, Gao M, Liu S, Li YQ, Song FH, et al. Intrathecal application of short interfering RNA knocks down c-jun expression and augments spinal motoneuron death after root avulsion in adult rats. Neuroscience 2013;241:268-79.

21. Zhou LH, Wu W. Survival of injured spinal motoneurons in adult rat upon treatment with glial cell linederived neurotrophic factor at 2 weeks but not at 4 weeks after root avulsion. J Neurotrauma 2006;23:9207.

22. Vanicky I, Urdzikova L, Saganova K, Cizkova D, Galik J. A simple and reproducible model of spinal cord injury induced by epidural balloon inflation in the rat. J Neurotrauma 2001;18:1399-407.

23. Basso DM, Beattie MS, Bresnahan JC. A sensitive and reliable locomotor rating scale for open field testing in rats. J Neurotrauma 1995;12:1-21.

24. Barnabe-Heider F, Frisen J. Stem cells for spinal cord repair. Cell Stem Cell 2008;3:16-24.

25. Grulova I, Slovinska L, Nagyova M, Cizek M, Cizkova D. The effect of hypothermia on sensory-motor function and tissue sparing after spinal cord injury. Spine J 2013;13:1881-91.

26. Ulndreaj A, Badner A, Fehlings MG. Promising neuroprotective strategies for traumatic spinal cord injury with a focus on the differential effects among anatomical levels of injury. F1000Res 2017;6:1907.

27. Xiong Y, Mahmood A, Chopp M. Emerging treatments for traumatic brain injury. Expert Opin Emerg Drugs 2009;14:67-84.

28. Kjell J, Olson L. Rat models of spinal cord injury: from pathology to potential therapies. Dis Model Mech 2016;9:1125-37.

29. Lei P, Li Y, Chen X, Yang S, Zhang J. Microarray based analysis of microRNA expression in rat cerebral cortex after traumatic brain injury. Brain Res 2009;1284:191201.

30. Redell JB, Liu Y, Dash PK. Traumatic brain injury alters expression of hippocampal microRNAs: potential regulators of multiple pathophysiological processes. J Neurosci Res 2009;87:1435-48.

31. Kwon BK, Casha S, Hurlbert RJ, Yong VW. Inflammatory and structural biomarkers in acute traumatic spinal cord injury. Clin Chem Lab Med 2011;49:425-33.

32. Yokobori S, Zhang Z, Moghieb A, Mondello S, Gajavelli S, Dietrich WD, et al. Acute diagnostic biomarkers for spinal cord injury: review of the literature and preliminary research report. World Neurosurg 2015; 83:867-78.

33. Kwon BK, Stammers AM, Belanger LM, Bernardo A, Chan D, Bishop CM, et al. Cerebrospinal fluid inflammatory cytokines and biomarkers of injury severity in acute human spinal cord injury. J Neurotrauma 2010; 27:669-82.

34. Lubieniecka JM, Streijger F, Lee JH, Stoynov N, Liu J, Mottus R, et al. Biomarkers for severity of spinal cord injury in the cerebrospinal fluid of rats. PLoS One 2011;6:e19247.

35. Dalkilic T, Fallah N, Noonan VK, Salimi Elizei S, Dong $\mathrm{K}$, Belanger L, et al. Predicting injury severity and neurological recovery after acute cervical spinal cord injury: a comparison of cerebrospinal fluid and magnetic resonance imaging biomarkers. J Neurotrauma 2018;35:435-45.

36. Sengupta MB, Basu M, Iswarari S, Mukhopadhyay KK, Sardar KP, Acharyya B, et al. CSF proteomics of secondary phase spinal cord injury in human subjects: perturbed molecular pathways post injury. PLoS One 2014;9:e110885.

37. de la Torre JC. Spinal cord injury: review of basic and applied research. Spine (Phila Pa 1976) 1981;6:315-35.

38. Cheriyan T, Ryan DJ, Weinreb JH, Cheriyan J, Paul JC, Lafage V, et al. Spinal cord injury models: a review. Spinal Cord 2014;52:588-95. 